\title{
Dysplastic Koilin Causing Proventricular Obstruction in an Eclectus Parrot (Eclectus roratus)
}

\author{
Ryan De Voe, DVM, Laurel Degernes, DVM, Dipl ABVP (Avian), and \\ Kristie Karli, DVM
}

\begin{abstract}
A 7-year-old male eclectus parrot (Eclectus roratus) was presented for evaluation of acute onset of dyspnea, tachypnea, and lethargy. Proventricular dilatation was observed on survey radiographs. Contrast fluoroscopic examination revealed decreased gastrointestinal motility and retention of contrast material in the proventriculus. Despite supportive care, the bird died. At necropsy, a tubular diverticulum of the ventriculus extended orad into the proventriculus. This diverticulum caused almost complete obstruction of proventricular outflow. No inflammation, infectious organisms, or neoplasia were associated with the lesion. The cause of the ventricular diverticulum in this parrot is unknown.
\end{abstract}

Key words: dysplastic koilin, gastrointestinal obstruction, contrast fluoroscopy, eclectus parrot, Eclectus roratus

\section{Introduction}

The ventriculus, or muscular stomach of birds, is lined on its mucosal surface by a cuticle layer, the koilin. The structure of both the ventriculus and the koilin varies according to diet between species of birds. The koilin serves as a grinding surface, enabling the ventriculus to mechanically digest food as it contracts. ${ }^{1}$ Insectivorous, herbivorous, and granivorous birds possess a well-developed, muscular ventriculus with a thick, abrasive koilin layer. In contrast, birds that eat foods not requiring significant mechanical digestion (ie, carnivores and piscivores) have an almost rudimentary ventriculus and soft koilin. ${ }^{1-4}$

Koilin consists of a carbohydrate-protein complex that is secreted from glands in the mucosa of the ventriculus. ${ }^{1-4}$ After this complex is secreted, it hardens in response to exposure to hydrochloric acid produced by the proventriculus. This process results in a tough, wear resistant material. ${ }^{1,2,4}$ Disruption of normal koilin architecture or production resulting in structural defects has been reported in many species of birds. ${ }^{5-17}$ Various etiologic factors

From the Departments of Clinical Sciences (De Voe, Degernes) and Population Health and Pathobiology (Karli), College of Veterinary Medicine, North Carolina State University, 4700 Hillsborough Street, Raleigh, NC 27606, USA. have been implicated in causing defects in the koilin layer, including a variety of infectious agents.

The structure of the koilin layer of the ventriculus is complicated. The basic structure consists of vertical rodlets of hard koilin that are produced by clustered tubular glands found in mucosal crypts. These rodlets collectively form the rods that are the vertical scaffolding of the koilin layer. The rods are surrounded by a horizontal matrix of desquamated cells and softer material produced by the surface epithelium. ${ }^{1}$ The koilin layer is continually worn down by grinding action and replaced by glandular secretion. ${ }^{1,2}$ In most birds with a well-developed ventriculus, the koilin is asymmetrically formed, being thickest opposite the craniodorsal and caudoventral thick muscles. ${ }^{1,2,4}$

In this report, we describe a ventricular diverticulum and koilin dysplasia of unknown cause in an eclectus parrot (Eclectus roratus) that resulted in gastrointestinal obstruction and death.

\section{Case Report}

A 7-year-old male eclectus parrot was examined because of dyspnea, tachypnea, and lethargy. The parrot was hand-raised and had been with the current owner for 1 year. It was housed in a room with two conures (Aratinga auricapilla and Nandayus nenday) and a female eclectus parrot, which had all 
been obtained at once from the same source. No abnormalities were reported with the other birds. The diet consisted of commercial pellets supplemented with seed, fresh fruits, and vegetables. All other husbandry practices were considered adequate. There was no known history of toxin exposure. The dyspnea and tachypnea had developed the previous day, and the owner had presented the bird to an emergency clinic late that evening. At the emergency clinic, it was placed in an oxygen cage and given intramuscular injections of enrofloxacin (Baytril, Bayer Corp, Shawnee Mission, KS, USA) and vitamin $\mathrm{A}, \mathrm{D}$, and $\mathrm{E}$. The next morning, the bird was transferred to our hospital for evaluation.

On examination, the bird was in good body condition and weighed $352 \mathrm{~g}$. The rhinotheca was moderately overgrown, and the choanal papillae were severely blunted. Feather loss from feather plucking was present over most of the body. The bird was tachypnic (approximately 60 breaths per minute) and moderately dyspneic at rest, with a tail-bob and an audible click on expiration. It was very lethargic and rested with its beak on the ground to help support its weight. The coelom was mildly distended. Dehydration was estimated at $8-10 \%$.

Jugular venipuncture was performed to obtain a blood sample for a complete blood count (CBC) and a plasma biochemical analysis. Results of the CBC revealed a mildly increased packed cell volume (56\%; reference range $45-55 \%) .{ }^{18}$ Results of the biochemical analysis revealed high concentrations of aspartate aminotransferase (555 IU/L; reference range, 135-230 IU/L), creatine kinase (4277 IU/L; reference range, 200-625 IU/L), and lactate dehydrogenase (944 IU/L; reference range, 100-280 IU/ L). ${ }^{18}$ All other CBC and plasma biochemical values were within reference ranges. Results of a fecal Gram stain were unremarkable. A cloacal swab specimen was submitted for aerobic and fungal culture. No pathogens were isolated.

The bird became severely dyspneic after examination and sample collection, necessitating hospitalizing the bird in a warm oxygen cage. After the bird's condition stabilized, fluid, antibiotic, and antifungal therapy was begun. The treatment regimen consisted of enrofloxacin $(25 \mathrm{mg} / \mathrm{kg}$ SC q24h; Baytril, Bayer), itraconazole (10 mg/kg PO q12h; Sporanox, Janssen Pharmaceutica Products, Titusville, NJ, USA), and lactated Ringer's solution ( $50 \mathrm{ml} / \mathrm{kg}$ $\mathrm{SC}$ q12h). The bird was also fed $20 \mathrm{ml}$ of a nutritional supplement by gavage (Emeraid II, Lafeber Company, Cornell, IL, USA).

The next morning, the parrot's clinical condition appeared slightly improved. It had passed scant green feces and yellow urates, and it had eaten a small amount of seed overnight. To prevent struggling and dyspnea during handling, the bird was anesthetized with isoflurane to take whole-body survey radiographs. Radiographic abnormalities included a significantly dilated proventriculus $(\sim 2-3$ times the normal diameter) and loss of serosal detail in the caudal coelomic cavity (Fig 1).

Contrast fluoroscopy was then done to evaluate the coelomic structures and gastrointestinal motility. Barium sulfate was administered $(12 \mathrm{ml}, 1: 1$ barium and water) by gavage into the crop. The bird was placed in a closed, narrow cardboard box during imaging to prevent excessive movement. Standing lateral and dorsal-ventral images were taken at 20, 50, 110, and 200 minutes. Motility of the crop, proventriculus, and ventriculus motility was diminished. When the final images were taken at 200 minutes, only a small amount of contrast material had reached the ventriculus. A filling defect was apparent in the proventriculus.

With the exception of gavage feeding, previously prescribed treatments were continued after the initial survey radiographs and fluoroscopy. A small amount of regurgitated barium was observed in the cage that evening. The bird was found dead late that night.

At gross necropsy, abnormalities were restricted to the proventriculus and ventriculus. Grossly, a thin-walled conical diverticulum, approximately 2.5 $\mathrm{cm}$ long and $1.5 \mathrm{~cm}$ wide, protruded from the ventriculus into the proventriculus. Proventricular outflow was almost completely obstructed by this diverticulum. A small opening at the apex of the diverticulum allowed communication between the proventriculus and ventriculus. The ventriculus and diverticulum contained a moderate amount of grit, seed, and barium sulfate (Fig 2). Barium sulfate and a scant amount of seed were present in the proventriculus.

Histologically, the proventriculus and ventriculus were normal (Fig 3A). The ventricular diverticulum consisted of thin, layered muscle and sloughed mucosa. A layer of koilin was associated with this tissue but appeared abnormal. The koilin layer in the diverticulum was thinner than the layer within the ventriculus and lacked the regular columnated structure (Fig 3B). Marked hemorrhage and congestion of the esophageal serosa, consistent with blockage of the upper gastrointestinal tract, was present. No evidence of infectious disease, toxicosis, or pulmonary aspiration (barium sulfate or otherwise) was detected grossly or histologically. The diagnosis was ventricular diverticulum with dysplastic koilin resulting in gastrointestinal obstruction. 


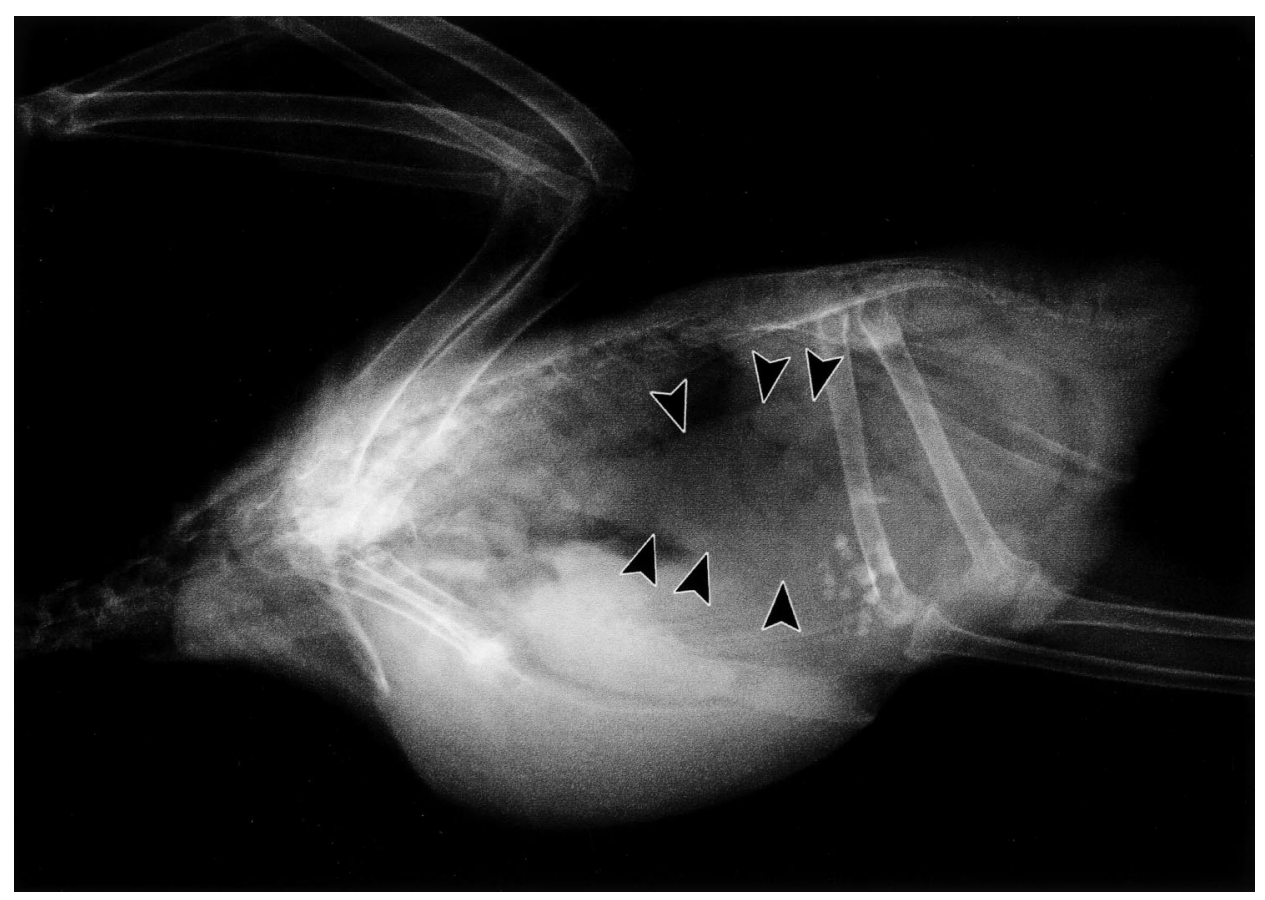

Figure 1. Lateral radiograph of an eclectus parrot with dysplastic koilin that resulted in obstruction of the proventriculus. The proventriculus is moderately enlarged (outlined by arrows).

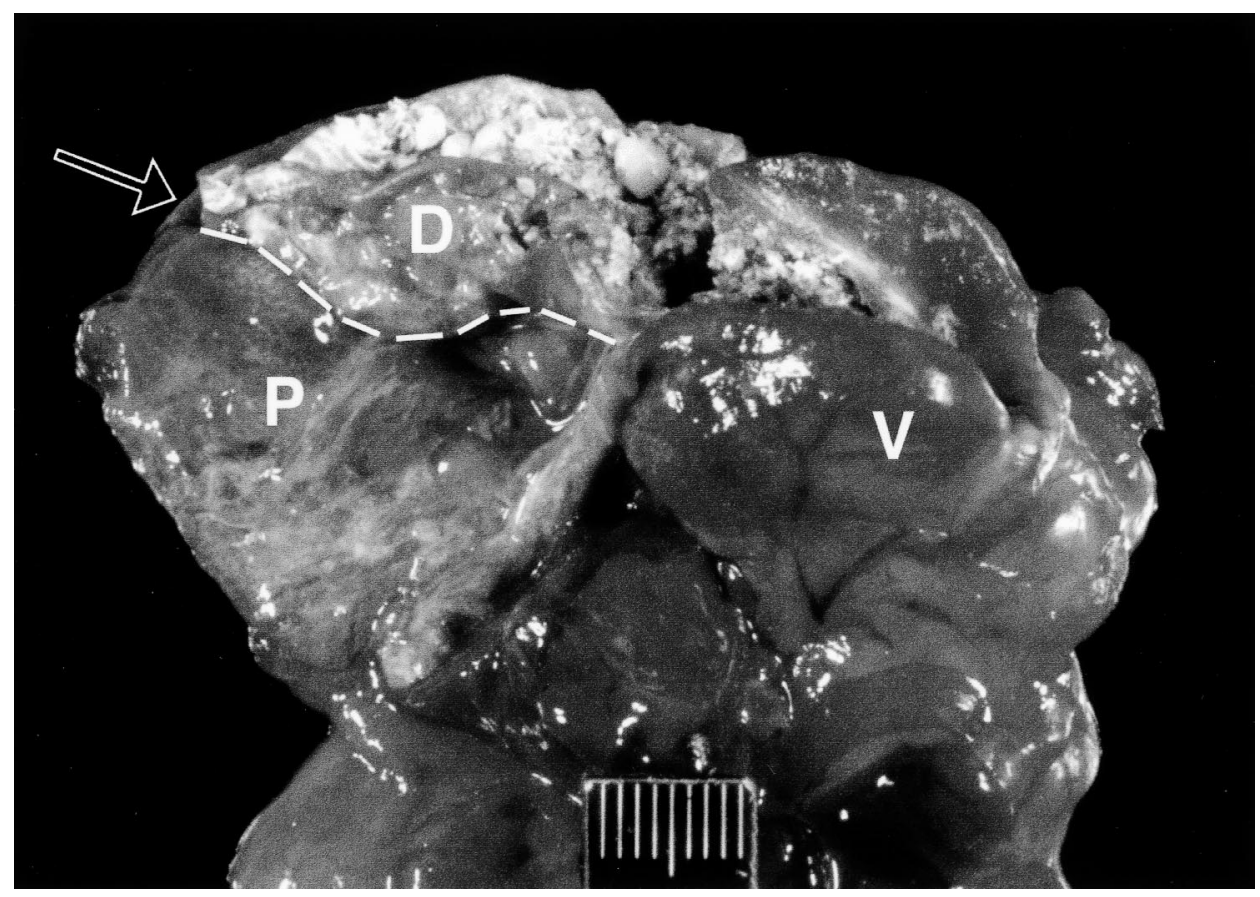

Figure 2. The proventriculus and ventriculus of the eclectus parrot described in Figure 1. The diverticulum is conical (outlined by the dashed line) and extends from the partially incised ventriculus into the opened proventriculus. A small opening is present at the apex of the diverticulum (arrow). Ingesta and barium sulfate are visible within the partially opened diverticulum and ventriculus. D indicates diverticulum; V, ventriculus; and P, proventriculus. 

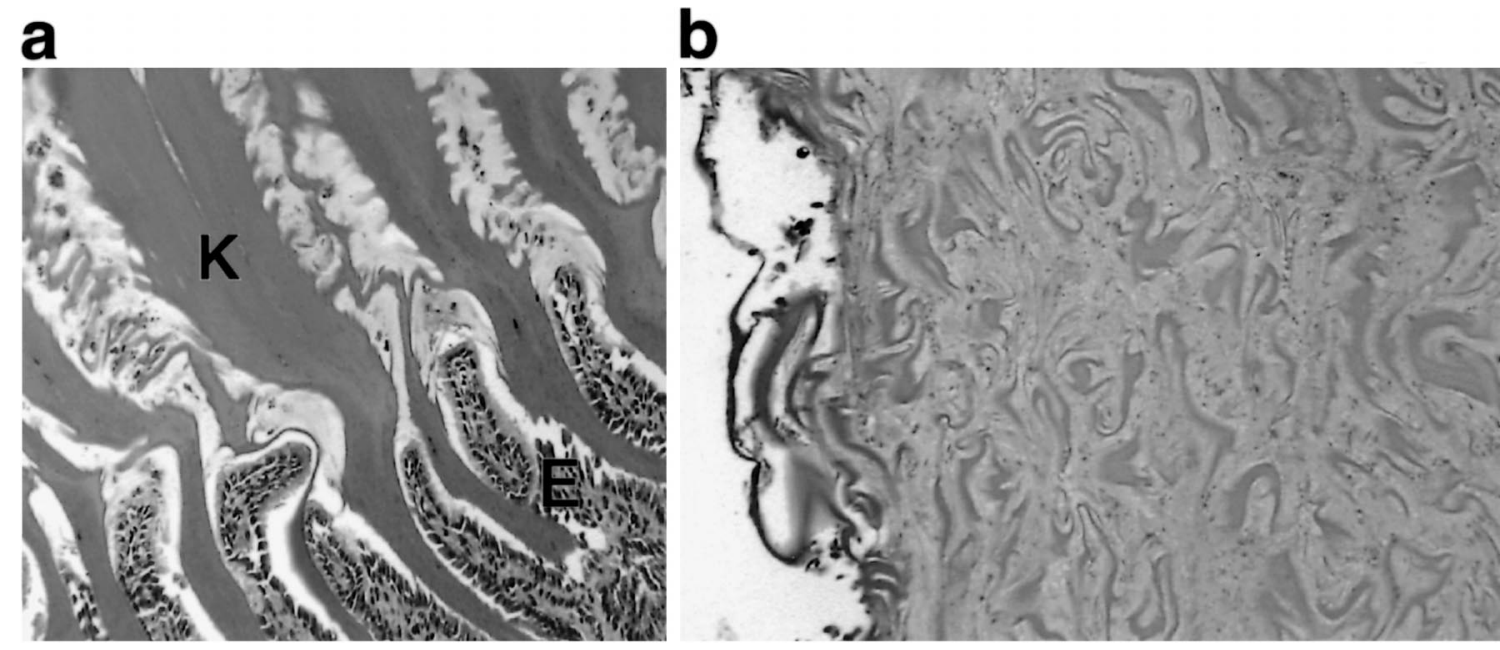

Figure 3. Photomicrograph of the normal ventricular koilin (a) and the dysplastic koilin of the ventricular diverticulum (b) from the eclectus parrot described in Figure 1. Note the regular, columnated structure of the normal koilin layer and its association with the glandular epithelium of the ventriculus. The koilin matrix of the diverticulum shows no distinct organization. K indicates koilin layer; E, glandular epithelium (hematoxylin and eosin $\times 400$ ).

\section{Discussion}

In this eclectus parrot, a diverticulum of the ventriculus caused almost complete obstruction of proventricular outflow, resulting in death. On histologic examination, the koilin layer of the diverticulum was thin, and the normal columnated structure was absent. No evidence of inflammation, infectious organisms, or neoplasia was found, and the cause of the ventricular diverticulum was not identified.

Gastrointestinal diseases are relatively common in avian species and can be challenging to diagnose. Infectious gastrointestinal diseases frequently occur and can be bacterial, fungal, viral, or parasitic in nature. Other common gastrointestinal disorders in avian patients include toxicosis, foreign body ingestion, papillomatosis, and neoplasia. ${ }^{19}$ Many clinical signs observed in birds with gastrointestinal disease are nonspecific, such as lethargy, anorexia, and a fluffed appearance. Illness can be acute or chronic. For these reasons, developing a specific list of differential diagnoses at presentation can be difficult.

In this parrot, the presenting complaints were dyspnea, tachypnea, and lethargy. The owner reported no regurgitation or vomiting, diarrhea, anorexia, weight loss, or other signs typically associated with gastrointestinal disease. ${ }^{20}$ This bird showed no overt signs of gastrointestinal disease initially, and gastrointestinal involvement was not suspected until the radiographic and fluoroscopic findings of proventricular dilatation, decreased proventricular and ventricular motility, and slow proventricular emptying of contrast material were iden- tified. Our initial differential diagnoses included primary respiratory disorders such as a tracheal or syringeal mass or foreign body, aspergillosis, chlamydiosis, sarcocystis, or an inhalant toxicosis (eg, polytetrafluoroethylene gas). ${ }^{21}$ Initial therapy was directed toward possible respiratory disease. Oxygen therapy, as well as administration of antimicrobials effective against most of the common respiratory pathogens, was instituted. In retrospect, the dyspnea and tachypnea seen in this bird were likely secondary to proventricular enlargement or associated discomfort. Respiratory difficulty is often observed in birds with organomegaly, as air sac volume is diminished. ${ }^{21}$

After identifying the radiographic and fluoroscopic abnormalities, gastrointestinal obstruction and proventricular dilatation disease were our primary differential diagnoses. Causes of partial to complete gastrointestinal obstruction in birds are foreign body ingestion, ${ }^{22,23}$ neoplasia, ${ }^{24-26}$ parasitic diseases, ${ }^{27}$ intussusception, ${ }^{28,29}$ stricture, abscesses or fungal granulomas, ${ }^{30,31}$ and sloughed koilin secondary to primary ventriculitis. ${ }^{32}$ Extraluminal masses impinging on the gastrointestinal tract can also result in obstructive conditions. ${ }^{31}$ Because movement of the barium was interrupted at the junction of the proventriculus and the ventriculus, an obstruction was strongly suspected. However, because the parrot had access to solid food until a few hours before contrast fluoroscopy, interpretation of the images was challenging. For this reason, we could not rule out the possibility that the apparent obstruction of the barium was caused by ingesta that had ac- 
cumulated secondary to functional ileus. Withholding solid food and feeding this bird a liquid diet for 24 hours or more before fluoroscopic evaluation may have been helpful. An acute obstruction was suggested by the fact that the bird was in good body condition and passing feces until less than 24 hours before its death.

Heavy metal analysis, especially for lead and zinc, would have been appropriate in this case but was not performed. Lead and zinc toxicosis have been associated with ileus and proventricular impaction. ${ }^{3}$ Zinc toxicosis associated with ventriculitis and erosions of the koilin has been reported in various avian species. ${ }^{10-12}$ Ingesting excessive amounts of other metals, such as copper, also has been associated with ventricular and koilin abnormalities. ${ }^{3}$

The koilin layer can be disrupted from a variety of causes. Mycotic proventriculitis or ventriculitis, such as that produced by avian gastric yeast or Candida species, can disturb koilin production.,5, $5,14,17$ Adenovirus infections in poultry have resulted in erosion of koilin layers. ${ }^{7,8}$ Other potential causes of koilin disruption are inappropriate diet (eg, hypovitaminosis A, excessive polyunsaturated fatty acids), ${ }^{13}$ parasitism, ${ }^{15}$ neoplasia, ${ }^{24-26}$ and toxicosis (eg, zinc, copper, ferrous sulfate)..$^{3,10-12,16}$ The koilin layer can also be eroded by ingestion of excessively abrasive objects or substances. ${ }^{13}$

The cause of the dysplastic koilin in this bird is unknown. Idiopathic intussusception of the proventriculus into the ventriculus has been described in chickens. ${ }^{33}$ However, the anatomic deformity observed in this eclectus parrot has not been previously described in other avian species. In this parrot, the ventricular diverticulum was intussuscepted into the proventriculus. In the absence of any inflammatory or malignant changes associated with the defect, the underlying cause was unlikely to be infectious or neoplastic. Possibly, a previous insult disrupted the koilin and mucosa of the ventriculus, initiating development of the diverticulum.

Normally, ingesta passes back and forth between the proventriculus and ventriculus, allowing combined chemical and mechanical digestion. ${ }^{34}$ In this bird, the strong contractions of the ventriculus might have progressively forced more and more ingesta into the diverticulum during normal reflux. The accumulated solid ingesta in the diverticulum eventually could have prevented the relatively weak contractions of the proventriculus from expelling the contents of the diverticulum into the ventriculus. By the time the diverticulum became clinically significant, the inciting cause might have resolved. Another possibility is that this ventricular diverticulum was a congenital anatomic defect that did not inter- fere with gastrointestinal function until it became distended with ingesta over time, ultimately causing a mechanical obstruction between the proventriculus and ventriculus.

This case illustrates the potential challenges of diagnosing gastrointestinal obstruction in avian patients. Also, structural abnormality involving the koilin should be considered as a differential diagnosis for gastrointestinal obstruction in psittacine birds.

\section{References}

1. King AS, McLelland J. Birds: Their Structure and Function. London, England: Bailliere Tindall; 1984: 84-109.

2. Akester AR. Structure of the glandular layer and koilin membrane in the gizzard of domestic fowl ( $\mathrm{Gal}$ lus gallus domesticus). J Anat. 1986;147:1-25.

3. Lumeij JT. Gastroenterology. In: Ritchie BW, Harrison GJ, Harrison LR, eds. Avian Medicine: Principles and Application. Lake Worth, FL: Wingers; 1994:483-521.

4. Denbow, MD. Alimentary canal: anatomy, regulation of feeding and motility. In: Whittow, GC, ed. Sturkie's Avian Physiology. 5th ed. New York, NY: Academic Press; 2000:299-321.

5. Jeffrey JS, Chin RP, Shivaprasad HL, et al. Proventriculits and ventriculitis associated with zygomycosis in ostrich chicks. Avian Dis. 1994;38:630-634.

6. Anderson, NL. Candida/megabacteria proventriculitis in a lesser sulphur-crested cockatoo (Cacatua sulphurea sulphurea). J Assoc Avian Vet. 1993;7:197-201.

7. Abe T, Nakamura K, Tojo T, Yuasa N. Gizzard erosion in broiler chicks by group 1 avian adenovirus. Avian Dis. 2001;45:234-9.

8. Goodwin MA. Adenovirus inclusion body ventriculitis in chickens and captive bobwhite quail (Colinus virginianus). Avian Dis. 1993;37:568-571.

9. Fosum O, Sandstedt K, Engstrom BE. Gizzard erosions as a cause of mortality in white leghorn chickens. Avian Pathol. 1988;17:519-525.

10. Zdiarski JM, Mattix M, Bush RM, Montali RJ. Zinc toxicosis in diving ducks. J Zoo Wildl Med. 1994;25: 438-445.

11. Romagnano A, Grindem CB, Degernes L, Mautino M. Treatment of a hyacinth macaw with zinc toxicity. J Avian Med Surg. 1995;9:185-189.

12. Droual R, Meteyer CU, Galey FD. Zinc toxicosis due to ingestion of a penny in a gray-headed chachalaca (Ortalis cinereiceps). Avian Dis. 1991;35:10071001.

13. Keymer IF. Disorders of the digestive system. In: Samour J, ed. Avian Medicine. Philadelphia, PA: Mosby; 2000:193-210.

14. Hubbard GB, Schmidt RE, Eisenbrandt DL, et al. Fungal infections of ventriculi in captive birds. $J$ Wildl Dis. 1985;21:25-28.

15. Sterner MC, Stackhouse L. Parasitic ulcerative ven- 
triculitis in mallards (Anas platyrhynchos). J Wild Dis. 1987;23:680-682.

16. Wallner-Pendleton DP, Froman P, Hedstrom O. Identification of ferrous sulfate toxicity in a commercial broiler flock. Avian Dis. 1986;30:430-432.

17. Prattis SM, Cioffee CJ, Reinhard G, Zaoutis TE. A retrospective study of disease and mortality in zebra finches. Lab Anim Sci. 1990;40:402-405.

18. Clubb SL, Schubot RM, Joyner K, et al. Hematologic and serum biochemical reference intervals in juvenile eclectus parrots (Eclectus roratus). J Assoc Avian Vet. 1990;4:218-225.

19. Morrisey JK. Gastrointestinal diseases of psittacine birds. Semin Avian Exotic Pet Med. 1999;8:66-74.

20. Rupley AE. Diagnostic techniques for gastrointestinal diseases of psittacines. Semin Avian Exotic Pet Med. 1999;8:51-65.

21. Rupley AE. Manual of Avian Practice. Philadelphia, PA: WB Saunders; 1997:55-90.

22. Speer BL. Chronic partial proventricular obstruction caused by multiple gastrointestinal foreign bodies in a juvenile umbrella cockatoo (Cacatua alba). J Avian Med Surg. 1998;12:271-275.

23. Adamcak A, Hess LR, Quesenberry KE. Intestinal string foreign body in an adult umbrella cockatoo (Cacatua alba). J Avian Med Surg. 2000;14:257263.

24. Schmidt RE, Dustin LR, Slevin RW. Proventricular adenocarcinoma in a budgerigar (Melopsittacus un- dulatus) and a green-cheeked parakeet (Brotogeris pyrrhopterus). AAV Today. 1988;2:140-142.

25. Leach MW, Paul-Murphy J, Lowenstine LJ. Three cases of gastric neoplasia in psittacines. Avian Dis. 1989;33:204-210.

26. Campbell TW, Turner O. Carcinoma of the ventriculus with metastasis to the lungs in a sulphur-crested cockatoo (Cacatua galerita). J Avian Med Surg. 1999;13:265-268.

27. Wilson GH, Greenacre CB, Howerth EW, et al. Ascaridiosis in a group of psittacine birds. J Avian Med Surg. 1999;13:32-39.

28. Greenwood AG, Storm J. Intestinal intussusception in two red-tailed hawks (Buteo jamaicensis). Vet Rec. 1994;134:578-579.

29. Frasca S, Khan MI. Multiple intussusceptions in a juvenile rhea (Rhea americana) with proventricular impaction. Avian Dis. 1997;41:475-480.

30. McMillan MC. Avian gastrointestinal radiography. Comp Contin Educ Pract Vet. 1983;5:273-278.

31. McMillan MC. Imaging techniques. In: Ritchie BW, Harrison GJ, Harrison LR, eds. Avian Medicine: Principles and Application. Lake Worth, FL: Wingers; 1994:246-326.

32. Van Sant F. Zinc toxicosis in a hyacinth macaw. Proc Annu Conf Assoc Avian Vet. 1991:255-259.

33. Sharma UK. Intussusception of the proventriculus of chickens. Avian Dis. 1972;16:453-457.

34. Dziuk HE, Duke GE. Cineradiographic studies of gastric motility in turkeys. Am J Physiol. 1972;222: 159-166. 